\title{
First Polarized Proton Collisions at RHIC
}

\author{
T. Roser*, L. Ahrens*, J. Alessi*, M. Bai*, J. Beebe-Wang*, J.M. Brennan*, \\ K.A. Brown*, G. Bunce* , P. Cameron*, E.D. Courant*, A. Drees*, W. \\ Fischer*, R. Fliller III*, W. Glenn*, H. Huang*, A.U. Luccio*, W.W. \\ MacKay*, Y. Makdisi*, C. Montag*, F. Pilat*, V. Ptitsyn*, T. Satogata*, S. \\ Tepikian*, D. Trbojevic*, N. Tsoupas*, J. van Zeijts*, A. Zelenski*, K. \\ Zeno $^{*}$, A. Deshpande ${ }^{\dagger}$, K. Kurita ${ }^{\dagger}$, K. Krueger ${ }^{* *}$, H. Spinka ${ }^{* *}$, D. \\ Underwood $^{* *}$, M. Syphers ${ }^{\ddagger}$, I. Alekseev ${ }^{\S}$, D. Svirida ${ }^{\S}$, V. Ranjbar" ${ }^{\llbracket}$ J. Tojoll, \\ O. Jinnouchi ${ }^{\dagger \dagger}$, M. Okamura ${ }^{\dagger \dagger}$ and N. Saito ${ }^{\dagger \dagger \mid l}$

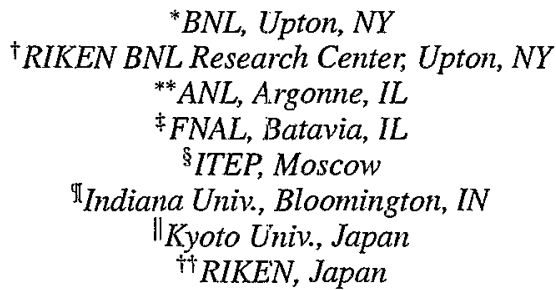

\begin{abstract}
We successfully injected polarized protons in both RHIC rings and maintained polarization during acceleration up to $100 \mathrm{GeV}$ per ring using two Siberian snakes in each ring. Each snake consists of four helical superconducting dipoles which rotate the polarization by $180^{\circ}$ about a horizontal axis. This is the first time that polarized protons have been accelerated to $100 \mathrm{GeV}$.
\end{abstract}

\section{INTRODUCTION}

Polarized proton colliders will open up the completely unique physics opportunities of studying spin effects in hadronic reactions at high-luminosity high-energy proton-proton collisions. It will allow study of the spin structure of the proton, in particular the degree of polarization of the gluons and antiquarks, and also verification of the many welldocumented expectations of spin effects in perturbative $\mathrm{QCD}$ and parity violation in $\mathrm{W}$ and $\mathrm{Z}$ production.

The Brookhaven Relativistic Heavy Ion Collider (RHIC) is the first hadron accelerator and collider consisting of two independent rings. It is designed to operate at high collision luminosity over a wide range of beam energies and with particle species ranging from polarized protons to heavy ions. The RHIC center-of-mass energy range of 200 to $500 \mathrm{GeV}[1]$ is ideal in the sense that it is high enough for perturbative QCD to be applicable and low enough so that the typical momentum fraction of the valence quarks is about 0.1 or larger. This guarantees significant levels of parton polarization.

\footnotetext{
${ }^{1}$ Work performed under the auspices of the U.S. Department of Energy
} 


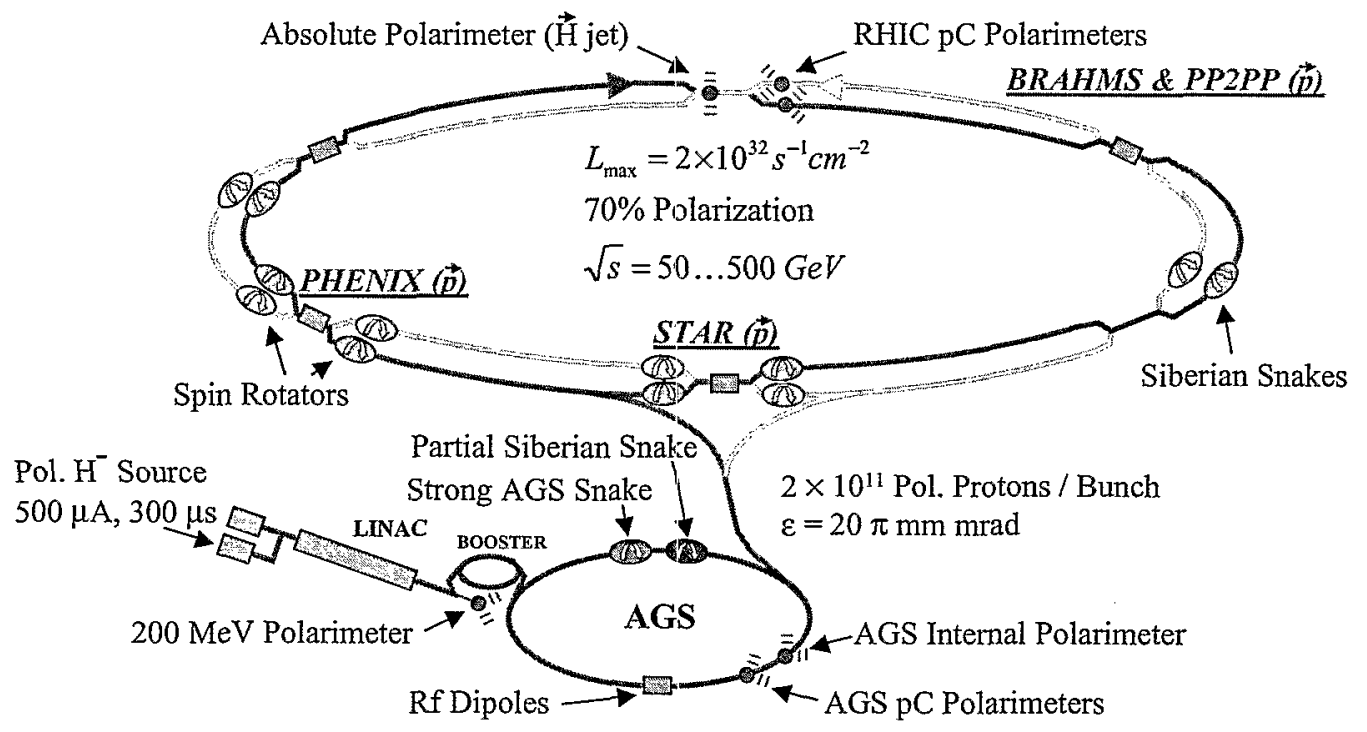

FIGURE 1. Layout and design parameters for the Brookhaven polarized proton collider. The eight spin rotators and the absolute polarimeter were not installed for this run. The beam parameters given correspond to the goal luminosity of $2 \times 10^{32} \mathrm{~cm}^{-2} \mathrm{~s}^{-1}$

During the second RHIC run polarized proton beams were successfully accelerated to $100 \mathrm{GeV}$ and stored and collided with a peak luminosity of about $1.5 \times 10^{30} \mathrm{~cm}^{-2} \mathrm{~s}^{-1}$. With the two full Siberian Snakes installed in each of the two RHIC rings essentially all beam polarization was preserved during acceleration and beam storage in RHIC.

\section{SPIN DYNAMICS, RESONANCES AND SIBERIAN SNAKES}

Accelerating polarized beams requires the control of both the orbital motion and spin motion. The evolution of the spin direction of a beam of polarized protons in external magnetic fields, such as those existing in a circular accelerator, is governed by the Thomas-BMT equation [2],

$$
\frac{d \vec{P}}{d t}=-\left(\frac{e}{\gamma m}\right)\left[G \gamma \overrightarrow{B_{\perp}}+(1+G) \overrightarrow{B_{\|}}\right] \times \vec{P}
$$

where the polarization vector $P$ is expressed in the frame that moves and rotates with the particle's velocity. This simple precession equation is very similar to the Lorentz force equation:

$$
\frac{d \vec{v}}{d t}=-\left(\frac{e}{\gamma m}\right)\left[\overrightarrow{B_{\perp}}\right] \times \vec{v} .
$$

Comparison of these two equations readily shows that, in a purely vertical field, the spin rotates $G \gamma$ times faster than the orbital motion. Here $G=1.7928$ is the anomalous 
magnetic moment of the proton and $\gamma=E / m$. G gives the number of full spin precessions for every revolution and is also called the spin tune $v_{s p}$. At top RHIC energies $v_{s p}$ reaches about 450 .

The acceleration of polarized beams in circular accelerators is complicated by the presence of numerous depolarizing spin resonances. During acceleration, a spin resonance is crossed whenever the spin precession frequency equals the frequency with which spin-perturbing magnetic fields are encountered. There are two main types of spin resonances corresponding to the possible sources of such fields: imperfection resonances, which are driven by magnet errors and misalignments, and intrinsic resonances, driven by the focusing fields. The strengths of both types of resonances increases with beam energy.

The resonance condition for imperfection depolarizing resonances arise when $v_{s p}=$ $G \gamma=n$, where $n$ is an integer. Imperfection resonances are therefore separated by only $523 \mathrm{MeV}$ energy steps. The condition for intrinsic resonances is $v_{s p}=k P \pm v_{y}$, where $k$ is an integer, $v_{y}$ is the vertical betatron tune and $P$ is the superperiodicity.

With a localized spin rotator that rotates the spin by the angle $\delta$ about a horizontal axis the spin tune is given by

$$
\cos \left(\pi v_{s p}\right)=\cos (\pi G \gamma) \cos (\delta / 2)
$$

The spin tune can never reach an integer for any non-zero $\delta$ and therefore all imperfection resonances are avoided. A 'full Siberian snake'[3], which is a $180^{\circ}$ spin rotator, will make the spin tune a half-integer and energy independent. Therefore, neither imperfection nor intrinsic resonance conditions can ever be met. In the presence of strong resonances the spin rotation of the snake has to be much larger than the total spin rotation from the resonances.

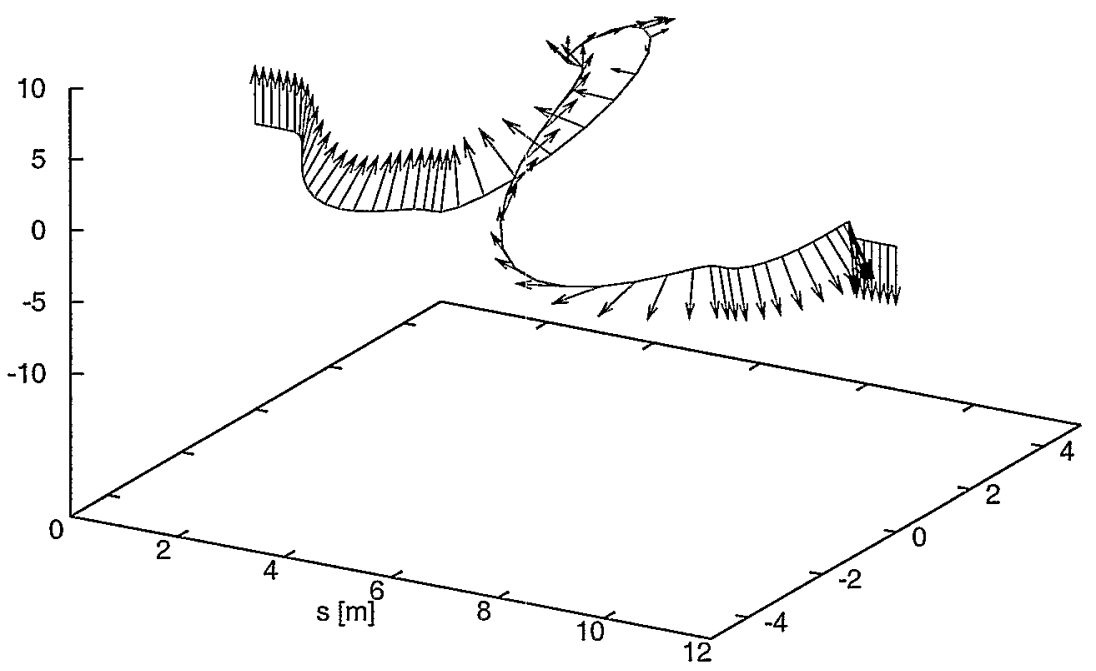

FIGURE 2. Orbit and spin tracking through the four helical magnets of a Siberian Snake at $\gamma=25$. The spin tracking shows the reversal of the vertical polarization. 


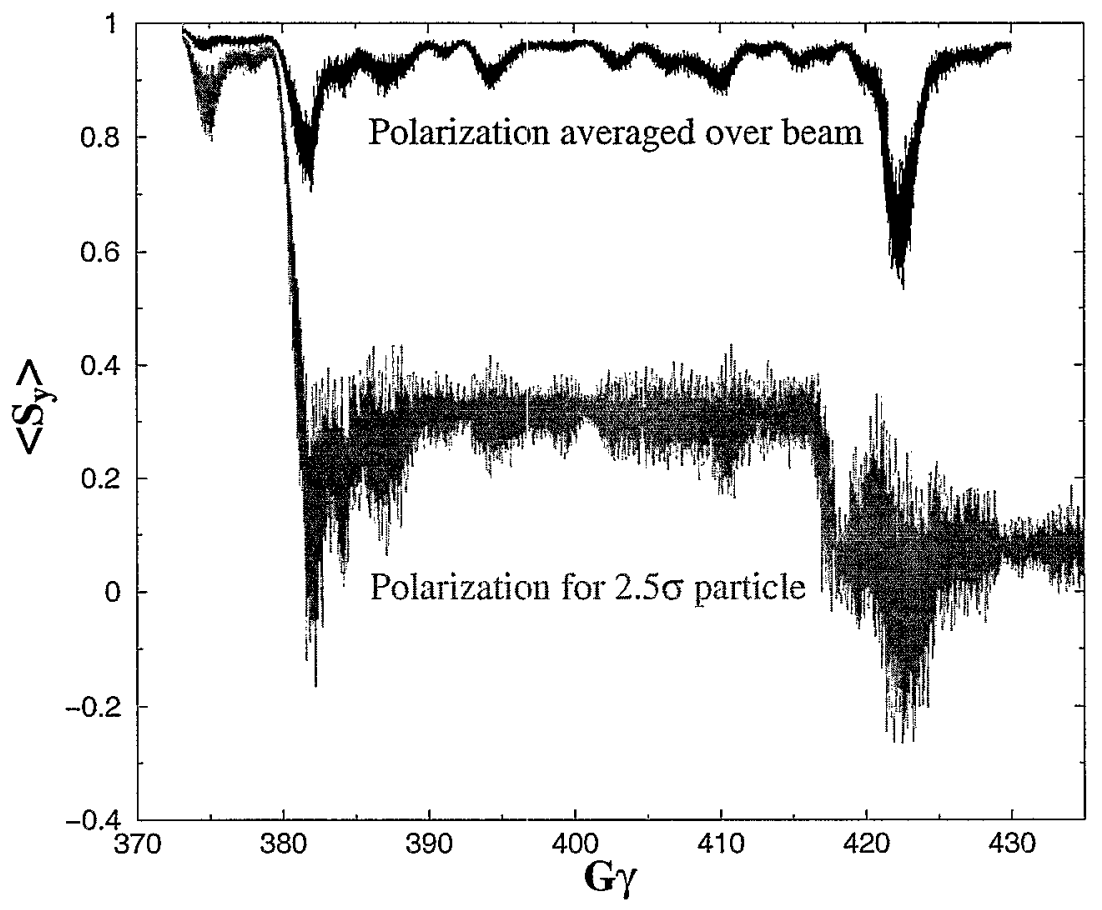

FIGURE 3. Result of spin tracking through the region of the strongest resonance in RHIC. The closed orbit was corrected with residuals of less than $0.2 \mathrm{~mm}$.

\section{ACCELERATING POLARIZED PROTONS IN AGS AND RHIC}

Fig. 1 shows the lay-out of the Brookhaven accelerator complex highlighting the components required for polarized beam acceleration. The new 'Optically Pumped Polarized Ion Source' (OPPIS)[4] produced $10^{12}$ polarized protons per pulse. A single source pulse is captured into a single bunch, which is ample beam intensity to reach the nominal RHIC bunch intensity of $2 \times 10^{11}$ polarized protons.

In the AGS a 5\% solenoidal partial snake that rotates the spin by $9^{\circ}$ is sufficient to avoid depolarization from imperfection resonances up to the required RHIC transfer energy of about $25 \mathrm{GeV}$ [5]. Full spin flip at the four strong intrinsic resonances can be achieved with a strong artificial if spin resonance excited coherently for the whole beam by driving large coherent vertical betatron oscillations[6]. The remaining polarization loss in the AGS is caused by coupling resonances and weak intrinsic resonances. Faster acceleration rate and a future, much stronger partial Snake should eliminate depolarization in the AGS[7].

The full Siberian snakes (two for each ring) and the spin rotators (four provide longitudinal polarization for an experiment) for RHIC each consist of four $2.4 \mathrm{~m}$ long, $4 \mathrm{~T}$ helical dipole magnet modules each having a full $360^{\circ}$ helical twist [8][9]. The $9 \mathrm{~cm}$ diameter bore of the helical magnets can accommodate $3 \mathrm{~cm}$ orbit excursions at injection. Fig. 2 shows the orbit and spin trajectory through a RHIC snake. The superconducting helical dipoles were constructed at BNL using thin cable placed into 


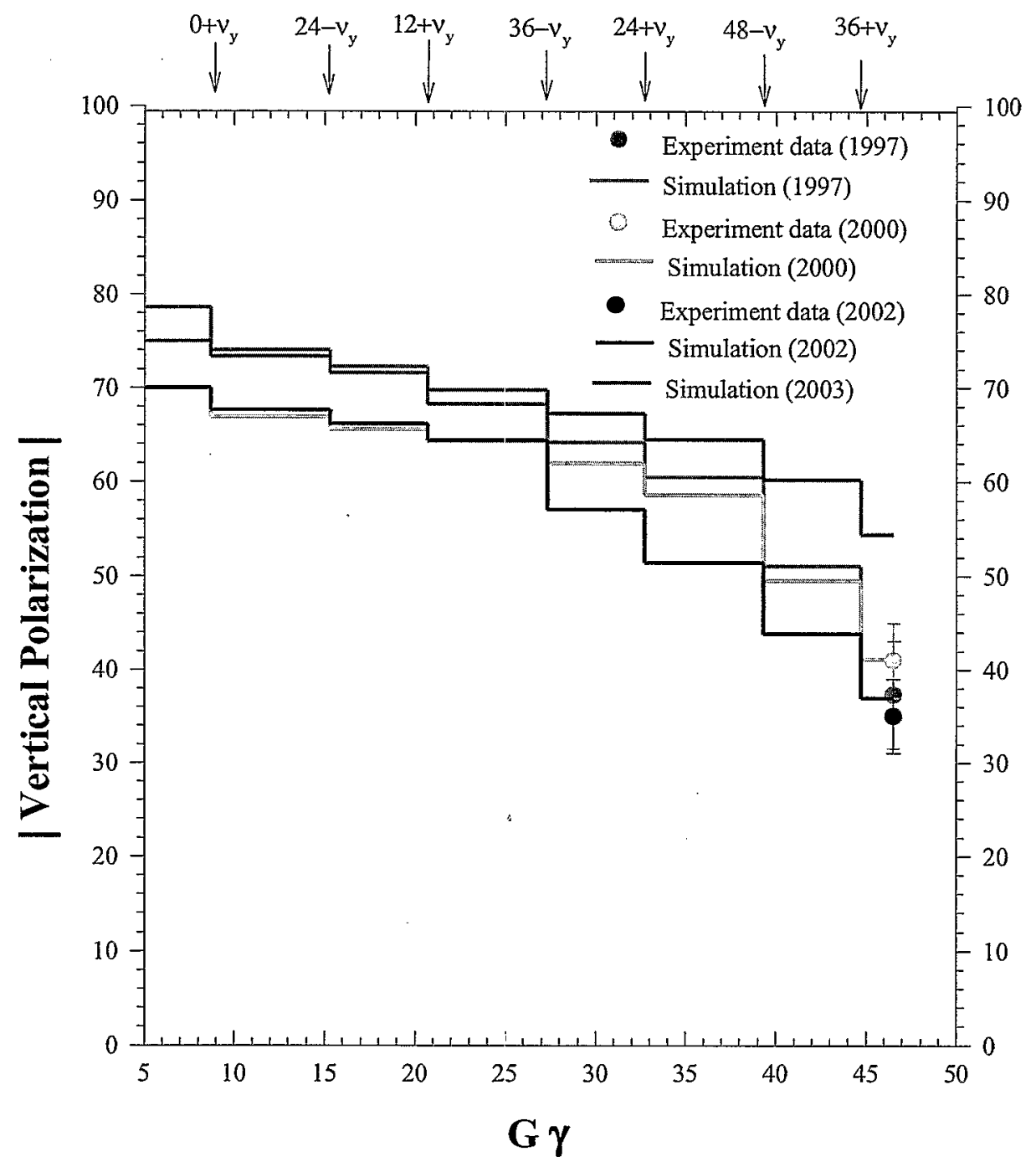

FIGURE 4. Beam polarization measured at the AGS extraction energy for recent running periods. Also shown are simulations of the polarization evolution during the acceleration ramp taking into account all intrinsic depolarizing resonances, the ramp rate, and coupling from the solenoidal partial Siberian snake.

helical grooves that have been milled into a thick-walled aluminum cylinder.

The acceleration through the energy region of the strongest resonance was simulated in great detail including a $1 \mathrm{~mm}$ rms misalignment of the quadrupoles, and sextupoles as well as the corrector dipoles used to correct the closed orbit. The result is shown in Fig. 3 for a beam with a normalized $95 \%$ emittance of $20 \pi \mathrm{mm}$ mrad. Although there is a significant decrease of the polarization at the energy of the strong resonance, the polarization of the full beam is restored after accelerating completely through the resonance region. The simulation also shows that there is significant polarization loss at 


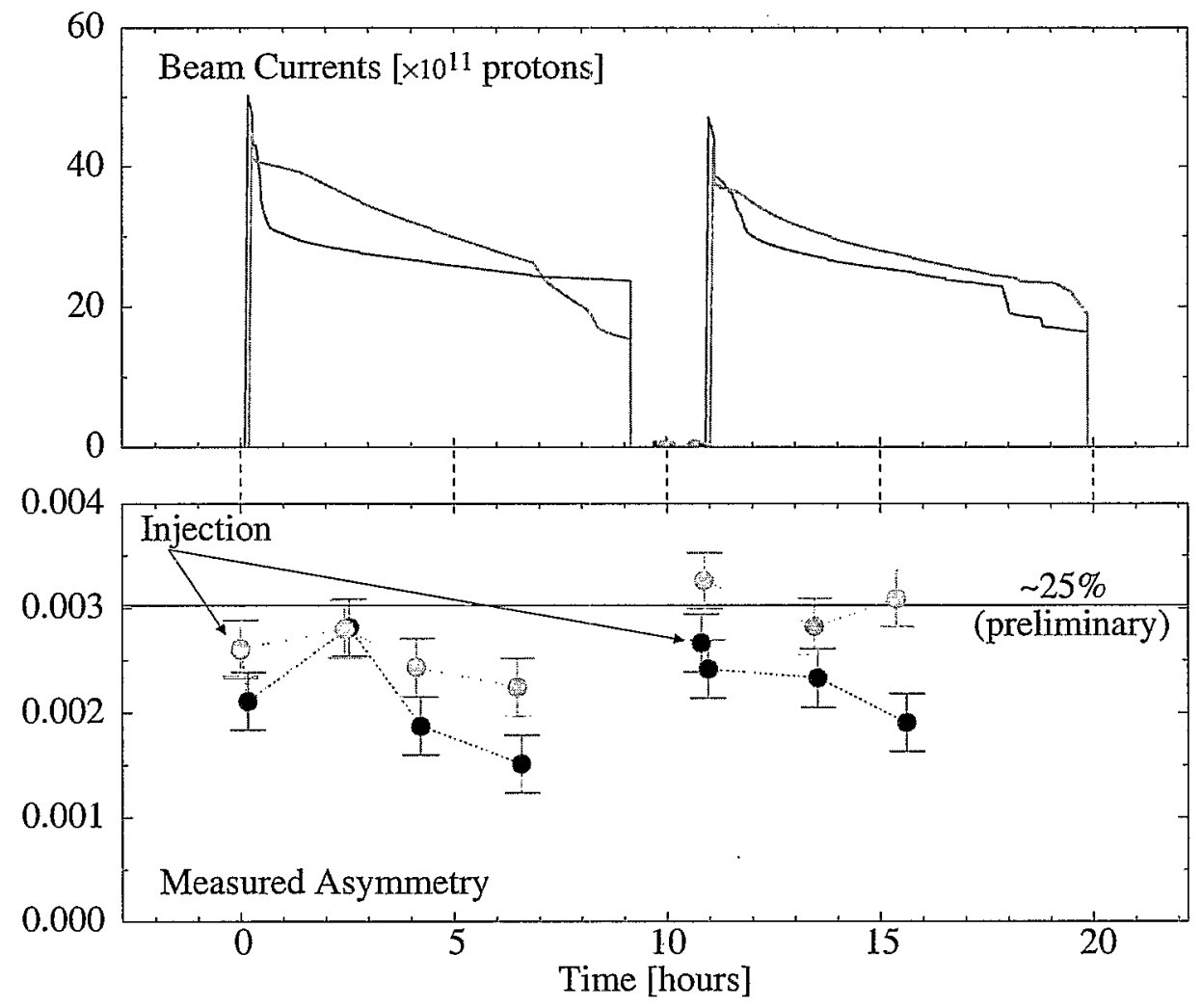

FIGURE 5. Circulating beam and measured asymmetry in the blue and yellow RHIC ring (blue(dark) and yellow(light) lines and symbols, respectively) for two typical stores.

the edge of the beam.

In addition to maintaining polarization, the accurate measurement of the beam polarization is of great importance. Very small angle elastic scattering in the CoulombNuclear interference region offers the possibility for an analyzing reaction with a high figure-of-merit which is not expected to be strongly energy dependent[10]. For polarized beam commissioning in RHIC an ultra-thin carbon ribbon was used as an internal target, and the recoil carbon nuclei were detected to measure both vertical and radial polarization components. The detection of the recoil carbon with silicon detectors using both energy and time-of-flight information showed excellent particle identification. It was demonstrated that this polarimeter can be used to monitor polarization of high energy proton beams in an almost non-destructive manner and that the carbon fiber target could be scanned through the circulating beam to measure polarization profiles.

\section{FIRST RHIC POLARIZED PROTON RUN}

The first polarized proton collider run in RHIC took place from Dec. 2001 to Jan. 2002. Polarized beams were successfully accelerated to $100 \mathrm{GeV}$ and stored and collided with 


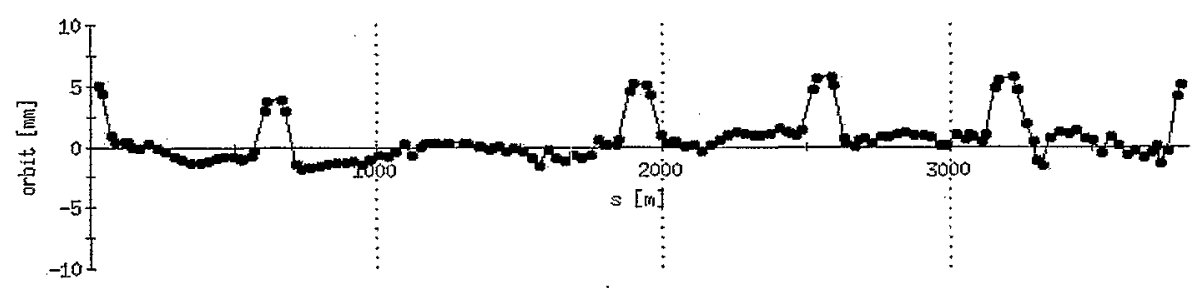

FIGURE 6. Typical vertical closed beam orbit in RHIC during the polarized proton acceleration cycle.

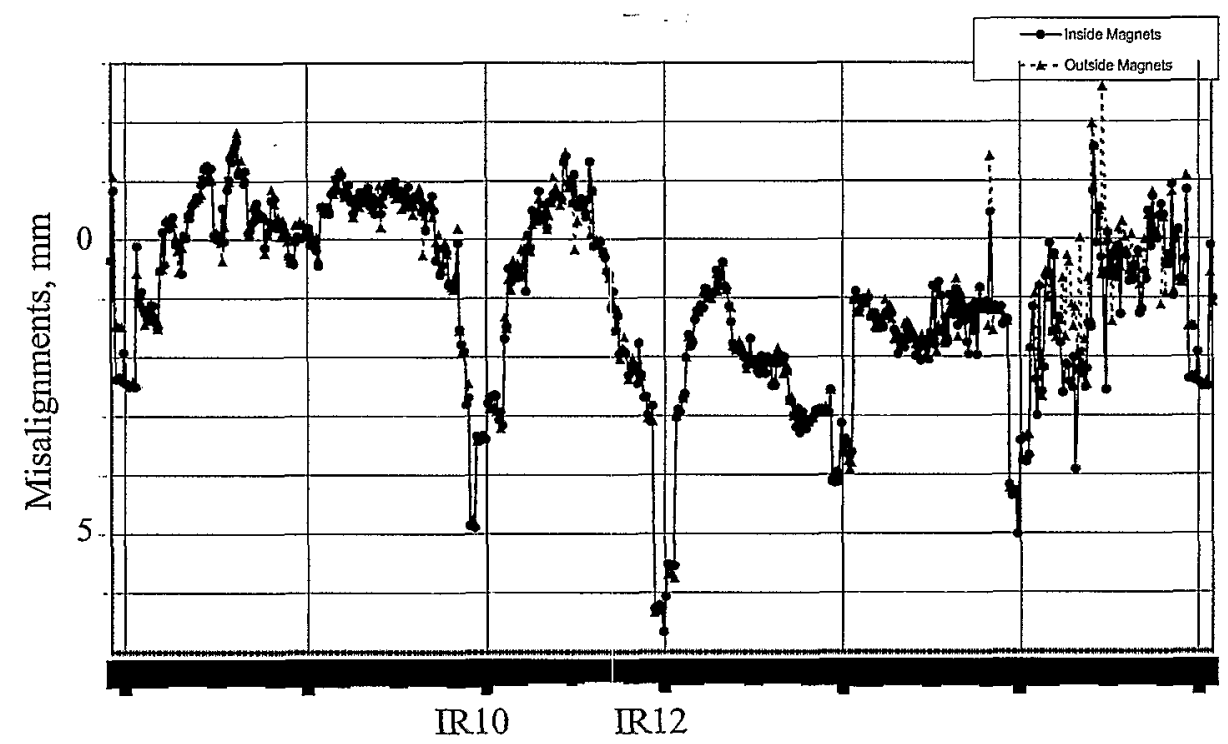

FIGURE 7. Vertical misalignments of the RHIC beam position monitors and quadrupoles

a peak luminosity of about $1.8 \times 10^{30} \mathrm{~cm}^{-2} \mathrm{~s}^{-1}$.

The beam polarization at the AGS was only about $30 \%$ mainly due to the fact that a back-up AGS main power supply had to be used with a much reduced ramp rate that amplified the effect of the weak intrinsic and the coupling depolarizing resonances. Fig. 4 shows the measured beam polarization at AGS extraction energy achieved at recent polarized proton runs. Also shown are simulation results that include the effect of the ramp rate and the coupling from the solenoidal partial Siberian snake on the strength of the intrinsic resonances in the AGS. The simulations are in reasonable agreement with the achieved polarizations. The simulation also predicts more than $50 \%$ polarization when the fast ramp-rate AGS main power supply is back in operation again.

In RHIC, essentially all beam polarization was preserved during acceleration and beam storage. Fig. 5 shows circulating beam current and measured asymmetries of two typical stores. The analyzing power at $100 \mathrm{GeV}$ for the RHIC polarimeters is not known but expected to be similar to the value at injection energy. Under this assumption the polarization at store was about $25 \%$. To preserve beam polarization in RHIC during 
acceleration and storage the vertical betatron tune had to be maintained between 0.220 and 0.235 and the orbit had to be corrected to better than $1 \mathrm{~mm} \mathrm{rms}$. This is in good agreement with the predictions from spin tracking calculations described above.

A typical vertical beam orbit in RHIC is shown in Fig. 6 . The beam excursions at the interaction regions serve to avoid beam collisions during acceleration. The remaining part of the orbit was adjusted to minimize vertical orbit excursions in the laboratory frame taking into account the absolute position of the RHIC beam position monitors. A new survey of the vertical alignment of the RHIC beam position monitors was completed after the end of the run and revealed quite large offsets up to about $5 \mathrm{~mm}$ as shown in Fig. 7. The effect of this offset on the RHIC beam polarization is still being analyzed, but it is clear that careful vertical realignment of the RHIC magnets and beam position monitors is necessary before acceleration of polarized beam to $250 \mathrm{GeV}$ is attempted. Beam-based orbit flattening could also be successful using vertical dispersion as an indication of vertical orbit errors.

More than 20 years after Y. Derbenev and A. Kodratenko made their proposal to use local spin rotators to stabilize polarized beams in high energy rings, it has now been demonstrated that their concept is working flawlessly even in the presence of strong spin resonances at high energy. Tests, verifying the Siberian Snake concept at low energy, were performed at IUCF[11].

All spin rotators will be installed for the 2003 run with the possibility to go to full collision energy of $\sqrt{s}=500 \mathrm{GeV}$ with longitudinal polarization at STAR and PHENIX. Finally a polarized gas jet will be installed for 2004 as an internal target for small angle proton-proton scattering which will allow the absolute calibration of the beam polarization to better than $5 \%$.

\section{REFERENCES}

1. Design Manual - Polarized Proton Collider at RHIC, Brookhaven National Laboratory, July 1998, http://www.rhichome.bnl.gov/RHIC/spin/design .

2. L.H. Thomas, Phil. Mag. 3, 1 (1927); V. Bargmann, L. Michel, V.L. Telegdi, Phys. Rev. Lett. 2, 435 (1959).

3. Ya.S. Derbenev et al., Part. Accel. 8, 115 (1978).

4. A.N. Zelenski et al., 'Optically-Pumped Polarized H- ION Sources for RHIC and HERA Colliders', Proc. of PAC99, N. Y., 106 (1999); A.N. Zelenski, these proceedings.

5. H. Huang et al., Phys. Rev. Lett. 73, 2982 (1994)

6. M. Bai et al., Phys. Rev. Lett. 80, 4673 (1998); M. Bai, these proceedings.

7. H. Huang, these proceedings.

8. V.I.Ptitsyn and Yu.M.Shatunov, NIM A398 (1997) 126

9. E. Willen et al., 'Construction of helical magnets for RHIC', Proc. of .PAC99, N. Y., .3161 (1999).

10. J. Tojo et al., Phys.Rev.Lett. 89, 052302 (2002); O. Jinnouchi et al., Spin2002 proceedings (BNL), to be published by AIP; G. Bunce, these proceedings.

11. A. D. Krisch et al., Phys. Rev. Lett. 63, 1137 (1989); J. E. Goodwin et al., Phys. Rev. Lett. 64, 2779 (1990) 